\title{
COMPARAÇÃO ENTRE MÉTODOS PARA DETERMINAÇÃo DE CARGAS ATÔMICAS EM SISTEMAS MOLECULARES: A MOLÉCULA N-\{N-(PTERINA-7-IL)CARBONILGLICIL\}-L-TIROSINA (NNPT)
}

\author{
Fernanda Diniz Botelho ${ }^{\mathrm{a}}$, Roberta Siqueira Soldaini de Oliveira ${ }^{\mathrm{a}}$, Joyce S. F. D. de Almeida ${ }^{\mathrm{a}}$, Tanos C. C. França, ${ }^{\mathrm{a}, \mathrm{b}, *, \odot}$ e \\ Itamar Borges Jr. a,\#,(i) \\ aDepartamento de Engenharia Química, Instituto Militar de Engenharia, 22290-270 Rio de Janeiro -RJ, Brasil \\ ${ }^{b}$ Department of Chemistry, Faculty of Science, University of Hradec Kralove, Rokitanskeho 62, 50003 Hradec Kralove, Czech \\ Republic
}

Recebido em 04/10/2020; aceito em 21/10/2020; publicado na web em 01/12/2020

\begin{abstract}
COMPARISON BETWEEN ATOMIC CHARGE METHODS FOR MOLECULAR SYSTEMS: THE N-\{N-(PTERIN-7-YL) CARBONYLGLYCYL\}-L-TYROSINE (NNPT) MOLECULE. Selecting a method to compute partial atomic charges is not trivial because different methods usually provide different charge values and there is no consensus on the most useful approach. In this work, Mulliken, MBS, Chelp, Chelpg, MK, Hirshfeld, NPA, DMA and AIM methods were selected to compute atomic charges and electric dipole moment vector of $\mathrm{N}-\{\mathrm{N}$-(Pterin-7-yl)carbonylglycyl $\}$-L-tyrosine molecule, a ricin inhibitor which has different types of bonds and chemical environments. While MBS and DMA methods provided the most chemically consistent charges according to atomic electronegativity and electron resonance effects criteria, Chelp, Chelpg and MK had the worst performances. Atomic charges and dipole moment calculated by the Hirshfeld method had the smallest magnitudes, a well-known behavior. Despite the differences among atomic charges predicted by all methods, the direction of the dipole moment vector was essentially the same. Further charge calculations using different basis sets and quantum methods indicated that the dependency on this aspect was the highest for Mulliken and Chelp and the lowest for MBS, Hirshfeld and DMA methods. Thus, results point to MBS and DMA as the most suitable methods for computing chemically consistent atomic charges and dipole moment vectors of similar systems for different applications; e.g., molecular dynamics.
\end{abstract}

Keywords: partial atomic charges; distributed multipole analysis, electric dipole moment vector; ricin inhibitors; computational chemistry.

\section{INTRODUÇÃO}

A densidade eletrônica que se acumula em torno de cada átomo de uma molécula é resultado da combinação de diversos fatores, tais como diferenças de eletronegatividade entre os átomos, carga total da molécula, efeitos de ressonância eletrônica, entre outros. A deficiência ou excesso de elétrons ao redor dos átomos está intimamente relacionada a propriedades químicas e físicas das moléculas, de modo que é essencial conhecer essa distribuição eletrônica para ser possível prever uma série de comportamentos da molécula de interesse. ${ }^{1,2}$

Métodos de cálculo de cargas atômicas particionam a densidade eletrônica entre os núcleos de uma molécula de maneira que a cada núcleo é associado um número, inteiro ou não, de elétrons. Este número, somado à carga elétrica positiva do correspondente núcleo, resulta na atribuição de cargas elétricas nominais a cada átomo, também conhecidas como cargas atômicas parciais. ${ }^{3-7} \mathrm{~A}$ atribuição de cargas atômicas parciais é essencial em simulações de dinâmica molecular, que utilizam esses valores para prever interações interatômicas e intermoleculares de repulsão ou de atração, além de poder ser utilizada na previsão de sítios reativos de uma molécula, na modelagem de sistemas solvatados, na comparação de propriedades químicas de sistemas semelhantes e, de maneira geral, para se modelar interações eletrostáticas de maneira quantitativa. ${ }^{5,8-10}$

Existem diversos métodos para determinar cargas atômicas parciais, que se diferenciam pela forma de se calcular tais cargas. De maneira geral, estes métodos podem ser divididos em três grupos. O primeiro engloba os métodos que particionam a função

*e-mail: tanos@ime.eb.br

"e-mail alternativo: itamar@ime.eb.br de onda molecular em termos das funções de base centradas nos átomos utilizadas para a expandir a função de onda, ou a densidade eletrônica, do sistema. Os métodos de Mulliken, ${ }^{11}$ Minimum Basis Set Mulliken (MBS) ${ }^{12}$ Natural Population Analysis (NPA) ${ }^{13}$ e Distributed Multipole Analysis (DMA) ${ }^{14}$ pertencem a este grupo.

No segundo grupo estão os métodos que dividem a densidade eletrônica em domínios atômicos no interior do espaço físico ocupado pela molécula. Esses domínios atômicos são definidos por meio do conceito de "átomos em moléculas" (AIM, sigla em inglês para atoms-in-molecules) de Bader $^{15}$ ou usam a densidade de uma "promolécula" que possui átomos neutros esfericamente simétricos localizados nas mesmas coordenadas dos átomos da molécula real, proposta por Hirshfeld; ${ }^{16}$ esta abordagem é a base do método que leva o nome deste autor.

O terceiro grupo de métodos reúne aqueles em que as cargas atômicas parciais são determinadas de maneira a reproduzir o potencial eletrostático molecular, tais como os métodos Charges from Electrostatic Potential (Chelp), ${ }^{17}$ Charges from Electrostatic Potential Grid based (Chelpg) ${ }^{18}$ e Merz-Kollman-Singh (MK). ${ }^{19}$ Esses três métodos diferem entre si na seleção dos pontos ao redor da molécula a serem utilizados no cálculo do potencial eletrostático molecular durante o processo de ajuste matemático., ${ }^{3,5,6}$

Como a carga elétrica de um átomo em uma molécula não é uma grandeza observável segundo a mecânica quântica, ela não pode ser medida. Por conseguinte, não é possível comparar os resultados das cargas calculadas com valores experimentais embora seja possível determiná-las indiretamente a partir da medida de observáveis fortemente dependentes das cargas, tais como o momento de dipolo e o gradiente de campo elétrico. ${ }^{20}$ Entretanto, embora não exista um consenso quanto ao melhor método para determinar cargas 
parciais, ${ }^{21,22}$ um conjunto de cargas atômicas calculado teoricamente deve possuir alguns requisitos, a saber: a soma das cargas de todos os átomos deve ser igual à carga total da molécula; os valores das cargas atômicas parciais devem ser consistentes com as eletronegatividades dos átomos e as cargas atômicas devem reproduzir o vetor momento de dipolo elétrico total da molécula de maneira acurada. 5, $5,21,23$

Embora o custo computacional de diferentes métodos de cálculo de cargas parciais atômicas varie, mesmo os mais eficientes computacionalmente podem ter seu uso restrito em sistemas contendo muitos átomos, por exemplo, em proteínas. Por essa razão, diversos campos de força, que são ferramentas da mecânica e dinâmica molecular usualmente utilizadas para o tratamento de sistemas contendo muitos átomos, empregam variados métodos para determinar cargas atômicas, muitos dos quais não derivados da química quântica. ${ }^{24}$ Os campos de força Chemistry at Harvard Macromolecular Mechanics (CHARMM) ${ }^{25}$ e diferentes gerações do Merck Molecular Force Field (MMFF) ${ }^{26}$ por exemplo, utilizam um modelo similar para o cálculo de cargas atômicas, a saber: a carga de um átomo parte de um valor inicial, que pode ser zero ou a carga atômica formal do átomo, e a esse valor são acrescentadas contribuições chamadas de incrementos ligação-carga, que refletem a polaridade da ligação química formada por esses átomos. Esses acréscimos são otimizados de maneira a reproduzir cargas elétricas de um conjunto de compostos utilizados como referência na parametrização do próprio campo de força, tratando-se, portanto, de métodos semiempíricos. Já os campos de força Optimized Potentials for Liquid Simulations (OPLS $)^{27-29}$ e Assisted Model Building with Energy Refinement (AMBER) ${ }^{30,31}$ utilizam métodos ab initio para determinar as cargas atômicas. As versões a partir de AMBER94 ${ }^{30}$ utilizam o algoritmo denominado Restrained electrostatic potential fit $(\text { RESP })^{32}$ para a derivação dessas cargas, sendo os cálculos realizados em fase gasosa, o que sistematicamente superestimava os momentos de dipolo. Esse problema foi abordado na versão AMBER03, ${ }^{31} \mathrm{em}$ que os cálculos são conduzidos em fase condensada com um modelo de solvente contínuo, ${ }^{33}$ o que, embora tenha representado um avanço na parametrização desse campo de força, não eliminou completamente as situações em que simulações computacionais não preveem resultados experimentais conhecidos. ${ }^{34}$

Neste trabalho, a molécula $\mathrm{N}-\{\mathrm{N}-($ Pterina-7-il)carbonilglicil $\}-\mathrm{L}-$ tirosina, ${ }^{35}$ aqui denominada NNPT (Figura 1), um inibidor da ricina, toxina encontrada nas sementes de mamona (Ricinus communis) ${ }^{36}$ foi utilizada para comparar diferentes métodos de cálculo de cargas atômicas parciais. Essa molécula contém ligações químicas distintas entre si quanto à polaridade e multiplicidade, anéis aromáticos contendo heteroátomos ou não e, ainda, diferentes grupos funcionais como amida, hidroxila e carboxila. Os nove métodos utilizados para calcular as cargas atômicas parciais dessa molécula foram Mulliken, ${ }^{11}$ MBS Mulliken, ${ }^{12}$ Chelp, ${ }^{17}$ Chelpg $,{ }^{18} \mathrm{MK},{ }^{19}$ Hirshfeld,,${ }^{16} \mathrm{NPA}^{13}$, DMA ${ }^{14}$ e AIM. ${ }^{15}$

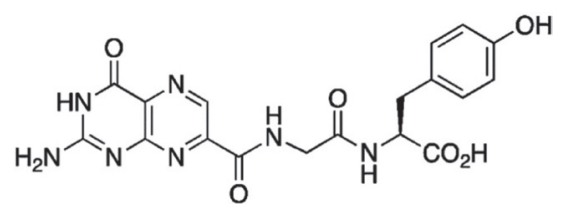

Figura 1. $N-\{N-($ Pterina-7-il)carbonilglicil $\}-L-t i r o s i n a(N N P T)$

\section{FUNDAMENTAÇÃO TEÓRICA}

\section{Métodos de determinação de cargas atômicas parciais}

O método de Mulliken ${ }^{11}$ é possivelmente o mais utilizado para determinar cargas atômicas parciais, possivelmente em razão de sua simplicidade computacional e por estar presente nos arquivos de saída padrão de muitos programas de química quântica. Esse método se baseia na teoria dos orbitais moleculares, na qual um conjunto de orbitais moleculares é expandido em uma combinação linear de orbitais atômicos. Os coeficientes da expansão são determinados variacionalmente pelo método de Hartree-Fock e a população eletrônica é separada em contribuições associadas a cada orbital atômico. ${ }^{22}$ A carga parcial de Mulliken $\left(q_{k}\right)$ no átomo $k$ é calculada de acordo com a Equação 1,

$$
q_{k}=Z_{k}-N_{k}
$$

em que $Z_{k}$ é a carga nuclear do átomo $k$ e $N_{k}$ é o número de elétrons associado a esse átomo. Para o cálculo de $N_{k}$, o método de Mulliken considera que toda a densidade eletrônica associada a um único átomo está localizada no átomo onde dada função de base atômica está centrada. A densidade eletrônica remanescente é associada às integrais de sobreposição entre dois átomos adjacentes, e é distribuída igualmente entre os átomos envolvidos nessa sobreposição. Por conseguinte, na região de uma ligação química, a densidade eletrônica é dividida igualmente entre os dois átomos que formam a ligação, independentemente das eletronegatividades dos átomos envolvidos, o que é uma das grandes falhas desse método. Outro problema associado ao método de Mulliken é o fato de ele utilizar um conjunto de funções de base não ortogonal, o que pode levar a resultados indesejáveis. Por exemplo, seria possível dividir o número total de elétrons pelas funções de base atômicas e obter, como resultado, mais de dois ou menos de zero elétrons para cada orbital molecular, o que viola o Princípio da Exclusão de Pauli. ${ }^{4-6,22,37}$ Entretanto, as cargas de Mulliken ainda são bastante utilizadas por terem um custo computacional baixo e serem obtidas trivialmente após um cálculo Hartree-Fock. ${ }^{4,2}$

O método de Mulliken é bastante dependente do conjunto de funções de base usado. Em geral, a utilização de bases menores (desde que compatíveis com o número de orbitais dos átomos em estudo) leva a melhores resultados porque, assim, tem-se cada função de base associada a um centro atômico. Em vista disso, foi desenvolvido posteriormente o método MBS, que inicialmente projeta os orbitais em um conjunto de funções de base mínimo, ou seja, um que contém apenas o número de funções de base necessário para acomodar todos os elétrons de um átomo. Em seguida, o cálculo de cargas é realizado de acordo com o método de Mulliken acima descrito. ${ }^{12}$ Por conseguinte, o método MBS fornece um balanceamento melhor da partição das cargas entre os átomos e resolve algumas inconsistências apresentadas pelo método original de Mulliken. ${ }^{5,38}$

Com o intuito de superar as deficiências associadas ao método de Mulliken, foi proposto um modelo de cargas derivadas do potencial eletrostático molecular, definido como a força eletrostática que age sobre uma carga elétrica positiva de valor unitário em dada posição da molécula. Esse modelo baseia-se no cálculo de um conjunto de cargas atômicas pontuais de modo que estas representem o potencial eletrostático molecular quântico em um conjunto predefinido de pontos ao redor da molécula. As maneiras de se escolher esse conjunto de pontos ao redor da molécula resultaram em diferentes métodos pertencentes a esse grupo. Dentre eles, destacam-se os métodos Chelp, ${ }^{17}$ Chelpg $^{18}$ e MK. ${ }^{19}$

No método Chelp, o potencial eletrostático é determinado em uma quantidade selecionada de pontos ao redor da molécula. Esses pontos estão contidos em camadas esféricas separadas pela distância de $1 \AA$ uma da outra e localizados em catorze pontos simétricos ao redor de cada átomo. O número total de pontos é selecionado de modo a se considerar uma região a $3 \AA$ da superfície de Van der Waals (VDW) da molécula, e pontos localizados no interior do raio de VDW de qualquer átomo são descartados devido às grandes distorções causadas 
pela proximidade com o núcleo. Já no método Chelpg, as camadas de pontos não estão centradas nos átomos que constituem a molécula. Os pontos são selecionados de maneira que estejam regularmente espaçados, com distância máxima de 2,8 ̊̊ em relação à superfície de VDW da molécula. Assim como no método Chelp, pontos localizados no interior do raio de VDW de qualquer átomo são descartados. Dessa forma, os pontos formam uma camada relativamente homogênea ao redor da molécula na qual todas as regiões fora do raio molecular de VDW e dentro da distância de interação definida estão igualmente representados. Por último, o método MK utiliza pontos localizados em quatro camadas, localizadas às distâncias de 1,4, 1,6, 1,8 e 2,0 vezes os raios de VDW dos átomos constituintes da molécula. A densidade de pontos em cada camada varia entre 1 a 5 pontos por $\AA$ dependendo do tamanho da molécula, gerando assim um total de 200 a 300 pontos por molécula. Uma das principais diferenças entre esses três métodos é o fato de que cargas calculadas pelo método Chelp podem variar de acordo com a conformação espacial da molécula já que os pontos de cálculo de potencial eletrostático gerados têm distâncias fixas em relação à superfície molecular de VDW. Já os algoritmos mais sofisticados utilizados pelos métodos Chelpg e MK para distribuição de pontos corrigem em parte essa falha. De maneira geral, as cargas derivadas do potencial eletrostático geram resultados melhores que aquelas calculadas pelo método de Mulliken. ${ }^{6,21}$

No método de Bader, ${ }^{15,39}$ que permite definir cargas AIM, utiliza-se um esquema particular de partição do espaço entre os átomos para ser usado em cálculos posteriores de cargas atômicas: o volume atômico é definido como a região do espaço que inclui o núcleo e está contida dentro das superfícies de fluxo zero ao redor deste núcleo. Essas regiões de fluxo zero são chamadas de pontos críticos da ligação química na teoria de Bader, e ocorrem quando o gradiente de densidade eletrônica passa de um valor negativo para um valor positivo, o que é observado quando se vai de um núcleo a outro considerando-se dois átomos ligados quimicamente. Matematicamente, a superfície de fluxo zero é definida como a união de todos os pontos que satisfazem a Equação 2,

$$
\nabla \rho \cdot \mathbf{n}=0
$$

em que $\rho$ é a densidade eletrônica e $\mathbf{n}$ é o vetor unitário normal à superfície. A Equação 2 é verdadeira se o gradiente da densidade eletrônica for paralelo à superfície (e assim seria ortogonal ao vetor n) ou se esse gradiente for nulo, que é o caso dos pontos críticos. Assim, a carga AIM de um átomo $\left(q_{k}\right)$ é definida como a diferença entre a carga nuclear $\left(Z_{k}\right)$ e o número de elétrons contidos no volume delimitado por superfícies de fluxo zero (definida na integral sobre o volume $\Omega_{k}$ cercado pela superfície de fluxo zero do átomo $k$ ), conforme Equação 3,

$$
q_{k}=Z_{k}-\int_{\Omega_{k}} \rho(\mathbf{r}) d \mathbf{r}
$$

Embora partam de um formalismo matemático elegante, as cargas AIM podem falhar na predição de cargas atômicas, atribuindo, por exemplo, cargas positivas a carbonos saturados e cargas negativas aos hidrogênios ligados a eles em hidrocarbonetos, ${ }^{22}$ além de serem conhecidas por serem significativamente maiores em magnitude quando comparadas às cargas derivadas de outros métodos. ${ }^{6,40}$ Isso ocorre porque a carga atribuída a um átomo pode ser distribuída de maneira bastante heterogênea dentro do volume desse átomo, fator que não é considerado na Equação 3.

O método de Hirshfeld ${ }^{16}$ parte de uma "promolécula" que possui átomos neutros esfericamente simétricos localizados nas mesmas coordenadas dos átomos da molécula real em estudo. Nesse método, as cargas parciais atômicas são calculadas considerando-se que a densidade eletrônica molecular em um determinado ponto é compartilhada pelos átomos circundantes, de acordo com a distância entre esse ponto e o núcleo de cada átomo. A contribuição de cada átomo é ponderada considerando-se a densidade eletrônica do átomo isolado a uma distância do núcleo correspondente à distância entre o ponto na molécula e o núcleo desse átomo. A carga Hirshfeld de um átomo $A, q_{A}$, é então definida pela Equação 4,

$$
q_{A}=Z_{A}-\int \frac{\rho_{A}^{0}(\boldsymbol{r})}{\sum_{A} \rho_{A}^{0}(\boldsymbol{r})} \rho(\boldsymbol{r}) d \boldsymbol{r}
$$

em que $Z_{A}$ é o número atômico do átomo $A, \rho_{A}^{0}(\boldsymbol{r})$ é a densidade eletrônica calculada para o átomo isolado A e $\sum_{A} \rho_{A}^{0}(\boldsymbol{r})$ é a densidade promolecular, ou seja, a densidade de superposição de todos os átomos neutros esfericamente simétricos. Assim, o segundo termo da parte direita da equação representa a população eletrônica nos átomos, integrada em todo o espaço de densidade definido por esse método.

Embora esse método seja um avanço em relação aos métodos de Mulliken e aos derivados do potencial eletrostático por considerar a eletronegatividade dos átomos por meio do fator de ponderação, $\frac{\rho_{A}^{0}(\boldsymbol{r})}{\sum_{A} \rho_{A}^{0}(\boldsymbol{r})}$ na Equação 4, existe um certo consenso na literatura que indica que as cargas calculadas pelo método Hirshfeld são usualmente muito pequenas, próximas de zero. ${ }^{5,23,41}$ De acordo com Ayers, ${ }^{42}$ isso ocorre porque esse fator de ponderação é calculado de tal maneira que considera que o átomo na molécula é muito similar ao átomo isolado. Assim, os dois termos do lado direito da Equação 4 se tornam muito similares e a diferença entre eles, $q_{A}$, é praticamente zero. Por outro lado, há trabalhos recentes que concluíram que o método de Hirshfeld forneceu resultados satisfatórios na previsão de cargas de átomos em ligações covalentes, ${ }^{38,43}$ de energia de ligações de hidrogênio entre moléculas de metanol $^{18}$ e de predição de regiosseletividade em reações substituição eletrofílica aromática. ${ }^{44}$

$\mathrm{O}$ método $\mathrm{NPA}^{13}$ se baseia na expansão de um conjunto de orbitais atômicos naturais (NAOs) para uma dada molécula em um conjunto de base de orbitais atômicos arbitrário. O método NPA então representa as ocupações (elementos diagonais da matriz densidade de uma partícula) desses NAOs para o sistema de interesse. Como os NAOs formam um conjunto ortonormal, abrangendo completamente o espaço da base de orbitais (geralmente não ortogonais), as populações naturais são inerentemente positivas e a soma fornece corretamente o número de elétrons. Como os NAOs são intrínsecos à função de onda e não a uma escolha particular de bases de orbitais, o método NPA é menos sensível a variações do tamanho conjunto de base. Assim, esse método evita falhas características do método de Mulliken ao mesmo tempo em que retém vantagens deste último, como o baixo custo computacional. ${ }^{22}$

Por fim, o método $\mathrm{DMA}^{14}$ descreve a distribuição de cargas em termos de uma expansão de momentos de multipolos elétricos centrados em dados pontos da molécula. Esses pontos em geral são os átomos da molécula, embora isso não seja obrigatório. A densidade de carga é então dividida em uma soma de produtos de funções de base gaussianas centradas nos átomos, cujos coeficientes são determinados a partir da matriz de densidade de um elétron. Os termos de expansão do método DMA possuem uma interpretação química intuitiva: os termos de monopolo correspondem às cargas atômicas; os dipolos fornecem informação sobre separação de cargas, ou seja, de polaridade e os momentos de quadrupolo são os primeiros momentos eletrostáticos a incluírem contribuições de "densidade fora do plano", sendo portanto usualmente associados à deslocalização de elétrons ou a pares isolados..$^{45} \mathrm{O}$ método DMA tende a ser mais acurado que outros métodos de cálculo de cargas parciais principalmente para cálculos de energia de interação intermolecular, já que esse método 
não particiona a carga elétrica molecular apenas em cargas atômicas, mas também, nesse caso, em momentos de dipolo e quadrupolo elétricos. Outro aspecto que torna o método DMA vantajoso é o fato de ele ser independente da função de base utilizada. ${ }^{37}$ Diversas aplicações da partição da densidade molecular de carga em multipolos elétricos DMA foram feitas pelo grupo de um dos autores (I.B.J.), incluindo estudos de sensibilidade de explosivos, ${ }^{46-48}$ otimização do tamanho de clusters ${ }^{49}$ e catálise..$^{50,51}$

\section{METODOLOGIA E DETALHES COMPUTACIONAIS}

\section{Otimização de geometria}

Os cálculos de otimização de geometria e de frequências foram realizados com o programa Gaussian $09 .{ }^{52}$

A geometria do NNPT (Figura 1) foi otimizada com o método DFT/B3LYP ${ }^{53,54}$ com conjunto de base $6-31 \mathrm{G}(\mathrm{d}) .{ }^{55}$ A escolha do método e da base se baseou no tamanho da molécula, que possui 48 átomos, e em seus átomos constituintes. Como todos os átomos pesados do NNPT pertencem ao segundo período da tabela periódica, a utilização da função $d$ no conjunto de base 6-31G possibilita a consideração da contribuição de orbitais vazios d nos orbitais mais internos dos referidos átomos. O funcional de troca e correlação B3LYP é um dos mais consagrados de sua categoria e foi utilizado neste trabalho por se tratar de um método robusto e ao mesmo tempo mais leve computacionalmente que outros funcionais, em parte por se tratar de um método semi-empírico. ${ }^{56}$ Os comprimentos de ligação calculados por esse método quântico foram comparados com dados de referência disponíveis em literatura para ligações em contextos químicos semelhantes aos existentes no $\mathrm{NNPT}^{57}$ pois não foram encontrados dados experimentais de geometria desta molécula. Para avaliar a qualidade dos resultados obtidos para os 50 comprimentos de ligação existentes na molécula, foi calculada a diferença percentual definida como diferença entre cada comprimento de ligação calculado $\left(c_{\text {calc }}\right)$ e o respectivo valor de referência $\left(c_{\text {ref }}\right)$ em relação a $c_{\text {ref }}$, conforme a Equação 5. Em seguida, foi calculada a média aritmética dos módulos dessas diferenças relativas (Equação 6).

$$
\text { Diferença percentual }=\frac{c_{\text {calc }}-c_{r e f}}{c_{r e f}} \times 100 \%
$$

Média dos módulos das diferenças $=\frac{1}{50} \sum_{i=1}^{50} \frac{\left|c_{\text {calc }}-c_{\text {ref }}\right|}{c_{r e f}} \times 100 \%$
A molécula com geometria otimizada foi submetida ao programa Spartan $08^{58}$ para geração de dez mil confôrmeros e seleção dos quinze confôrmeros de menor energia de acordo com o método de mecânica molecular MMFF. ${ }^{26}$ A energia desses quinze confôrmeros foi então reavaliada pelo método DFT/B3LYP ${ }^{53,54}$ com conjunto de base $6-31 \mathrm{G}(\mathrm{d})^{55}$ para comparação com a energia associada ao confôrmero resultante da otimização de geometria. Foi selecionada a conformação de menor energia - essa geometria foi utilizada para determinar as cargas parciais atômicas.

\section{Determinação das cargas atômicas}

As cargas atômicas foram determinadas por nove diferentes métodos, a saber, Mulliken, ${ }^{11} \mathrm{MBS},{ }^{12}$ Chelp $,{ }^{17} \mathrm{Chelpg},{ }^{18} \mathrm{MK},{ }^{19}$ Hirshfeld,${ }^{16} \mathrm{NPA}^{13}$, DMA ${ }^{14}$ e AIM..${ }^{15}$ Os cálculos de cargas atômicas pelo método DMA foram realizados utilizando-se o software GDMA $2^{14}$ e os cálculos de cargas atômicas pelo método AIM foram realizados utilizando-se o software Multiwfn. ${ }^{59}$ Todos os outros métodos de cálculo de cargas foram realizados no software Gaussian 09. ${ }^{52}$ Em todos os cálculos de carga atômica realizados, as coordenadas e o módulo do vetor momento de dipolo elétrico total da molécula também foram determinados, e estes valores foram analisados e comparados.

\section{RESULTADOS E DISCUSSÃO}

\section{Otimização de geometria}

A geometria otimizada da molécula de NNPT, utilizada para os cálculos de cargas, com os átomos numerados, está representada na Figura 2.

O resultado da média dos módulos das diferenças da geometria otimizada DFT/B3LYP usando a Equação 6 foi de 1,25\%, resultado satisfatório para nossos propósitos. Detalhes dos comprimentos de ligação calculados e de referência se encontram na Tabela $1 \mathrm{~S}$ do Material Suplementar.

\section{Cargas parciais e vetores momentos de dipolo}

Todos os valores de cargas parciais estão apresentados em múltiplos da carga elétrica elementar $\left(e=1,602 \times 10^{-19} \mathrm{C}\right)$ e estão listados nas Tabelas 2S a 4S do Material Suplementar.

Inicialmente, foram examinados os cinco átomos da molécula que apresentaram as maiores variações de valores de carga parcial

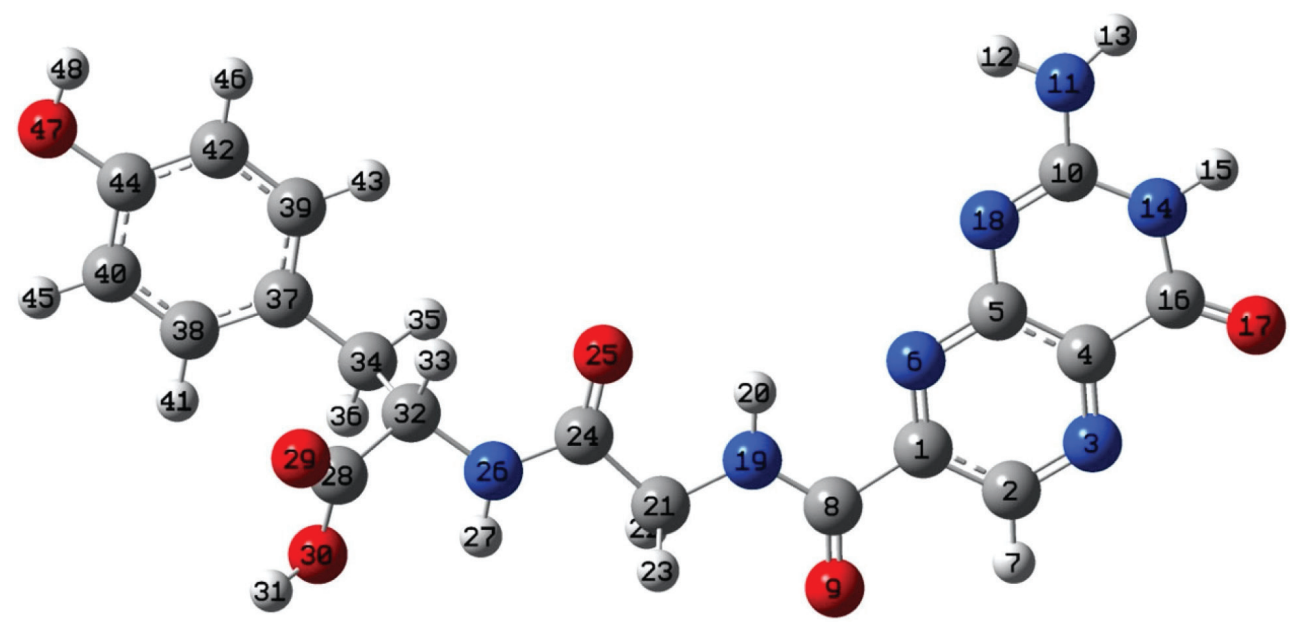

Figura 2. Geometria otimizada do NNPT. Átomos de carbono, hidrogênio, nitrogênio e oxigênio estão representados, respectivamente, pelas cores cinza, branco, azul e vermelho 
entre os métodos (Figura 3a) e os cinco átomos que apresentaram as menores variações desses valores (Figura $3 b$ ).

A Figura 3(a) mostra que os átomos que apresentaram as maiores variações de valores de cargas atômicas são todos átomos de carbono e, pela Figura 2, vê-se que todos têm em comum a característica de estarem ligados a pelo menos dois átomos mais eletronegativos (nitrogênio e/ou oxigênio). Todos os métodos, conforme esperado, apresentaram valores positivos para as cargas parciais desses átomos. Porém, as cargas AIM definidas pelo método de Bader apresentaram valores muito maiores (da ordem de $300 \%$ maiores que a média das cargas definidas pelos outros oito métodos) que as obtidas pelos outros métodos, provavelmente devido a uma supervalorização da diferença de eletronegatividade entre o átomo de carbono e os átomos de nitrogênio e oxigênio.

Os átomos cujas cargas menos variaram dentre os diferentes métodos estão indicados na Figura 3b. Todos são átomos pertencentes ao anel fenólico da molécula de NNPT, indicando uma boa concordância entre os diferentes métodos para previsão de cargas parciais de átomos de carbono e hidrogênio em anéis aromáticos derivados do benzeno. Os átomos C38 e C39 não só têm valores de cargas concordantes dentre os diferentes métodos como também possuem cargas elétricas muito similares entre si, o que ocorre por estarem em posições bastante parecidas na molécula. A maior parte dos métodos de cálculo de carga mostram que esses dois átomos têm carga parcial próxima de zero (os métodos Mulliken e NPA atribuíram cargas de maior módulo a esses átomos: $-0,1888$ e por Mulliken e $-0,2113$ e pelo NPA, ambas para o átomo $\mathrm{C} 38$ ). Os valores quase nulos das cargas dos átomos C38 e o C39 eram esperados já que esses dois átomos de carbono experimentam efeitos antagônicos. Por um lado, os átomos C38 e C39 estão, cada um, ligados a um átomo de hidrogênio, portanto tenderiam a concentrar em si uma carga parcial negativa. Entretanto, o grupo O47-H48 participa da ressonância eletrônica do anel benzênico e tende a concentrar a carga negativa nos átomos C37, C40 e C42 do anel, assim os átomos C38 e C39 tenderiam a ficar menos negativos que os três primeiros. Assim, a concentração de densidade eletrônica devido às ligações com átomos de hidrogênio é compensada pelos efeitos de ressonância eletrônica que retiram densidade eletrônica dos átomos C38 e C39, deixando-os com carga parcial próxima de zero.

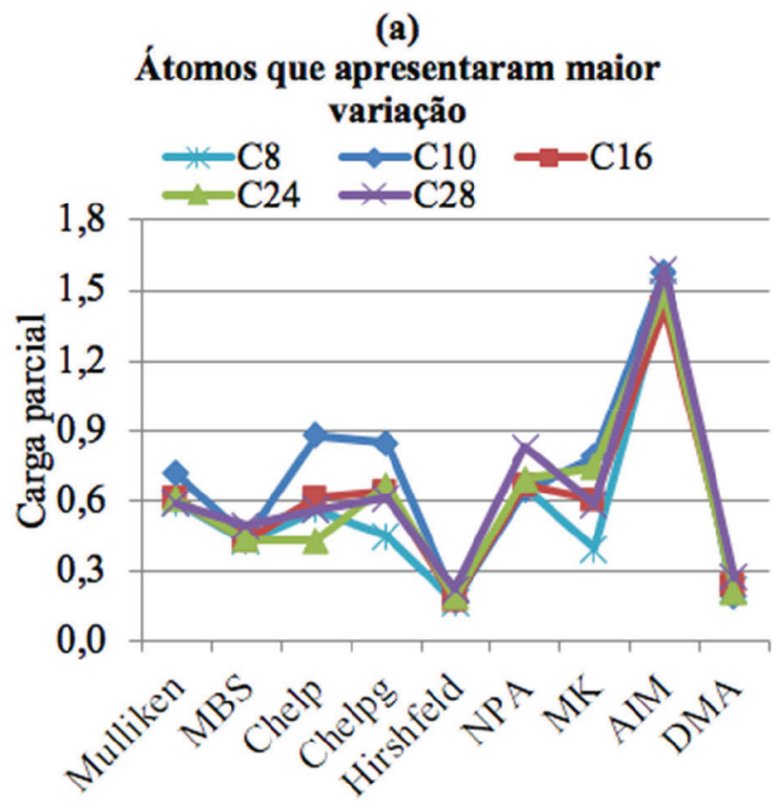

Os átomos H41, H43 e H46 também pertencem ao grupo de átomos que apresentaram menor variação de cargas atômicas calculadas por diferentes métodos - as curvas correspondentes a cada um desses átomos estão praticamente sobrepostas na Figura $3 b$, indicando que as cargas desses três átomos tendem a ser muito parecidas entre si. Para verificar as possíveis diferenças de cargas entre esses três átomos de hidrogênio, é necessário considerar os efeitos de ressonância provocados pelo átomo $\mathrm{O} 47$, sabendo-se que o grupo hidroxila formado por $\mathrm{O} 47$ e H48 é orto-para dirigente no anel benzênico. ${ }^{44,60}$ Assim, por ressonância eletrônica, o grupo O47-H48 tende a atribuir carga eletrônica negativa aos átomos C37, C40 e C42, portanto, espera-se que os átomos de hidrogênio ligados a esses carbonos tenham carga parcial menos positiva já que há uma tendência de concentração de cargas negativas próxima a eles. Dessa maneira, é esperado que H43, que está ligado a C39, seja mais positivo que H46 (ligado a C42), já que o primeiro está mais distante da concentração de cargas negativas. Esse resultado foi verificado para as cargas calculadas pelos métodos Mulliken, MBS, Chelpg, Hirshfeld, NPA, AIM e DMA. A rotação livre das ligações simples C34 - C37 e C44 - O47 e a simetria do anel fenólico indicam uma boa equivalência de posições entre os átomos $\mathrm{H} 41$ e H43, logo ambos deveriam ter cargas parciais de valores muito próximos entre si. Os métodos que apresentaram maior diferença relativa entre as cargas desses dois átomos foram Chelpg e AIM, cujas cargas dos átomos $\mathrm{H} 41$ têm um valor aproximadamente 30\% menor que as cargas dos átomos H43, para um mesmo método de cálculo.

Foram também examinadas as variações das cargas parciais dos átomos de oxigênio (Figura 4a) e de nitrogênio (Figura 4b) da molécula.

As cargas AIM dos átomos de oxigênio e nitrogênio são muito mais negativas que as cargas desses átomos calculadas por outros métodos, o que certamente influenciou na atribuição de cargas bastante positivas aos átomos de carbono da Figura 3(a), acima discutidas. Embora os átomos da Figura 4 sejam de fato os mais eletronegativos da molécula, não se espera que tenham cargas tão negativas quanto as cargas previstas pelo método AIM. As cargas AIM desses átomos são menores que - $e$, ou seja, cada átomo de nitrogênio ou oxigênio teria propriedades de um ânion monovalente, o que não é compatível com o comportamento de sistemas moleculares como a NNPT.

Comparando-se os átomos de oxigênio O9 e O25, pertencentes a grupos amida em posições similares na molécula, espera-se que

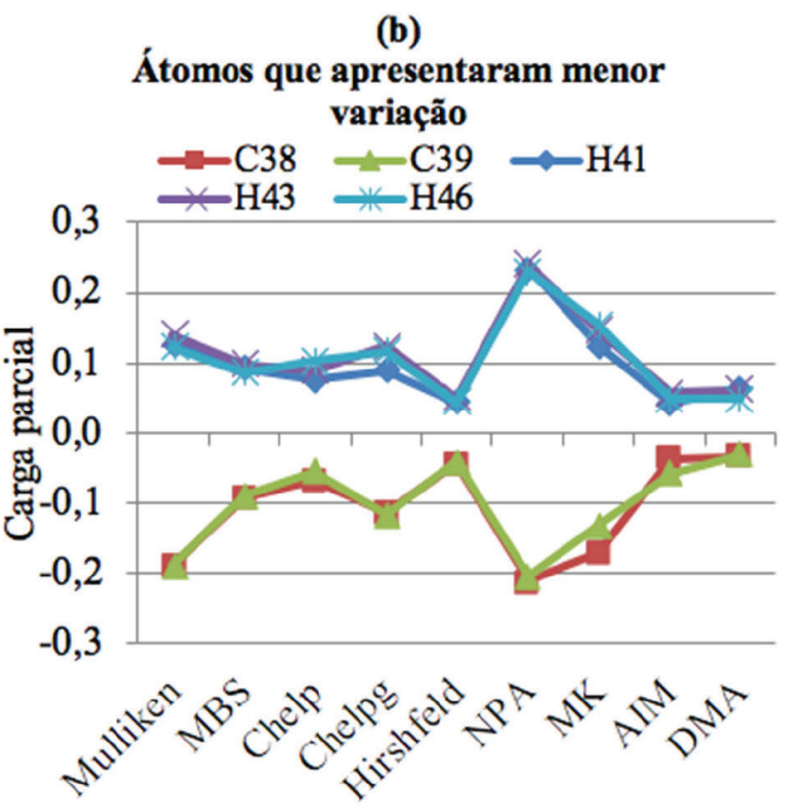

Figura 3. Cargas atômicas calculadas pelos nove diferentes métodos em unidades de e. (a) Cinco átomos que apresentaram maior variação de valores calculados pelos métodos; (b) Cinco átomos que apresentaram menor variação de valores calculados pelos métodos 
(a) Variação das cargas dos átomos de
oxigênio
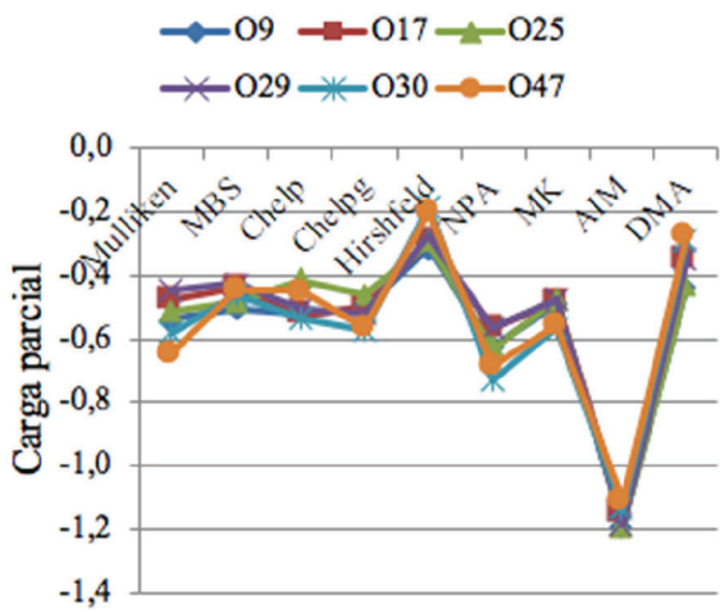

(b)

Variação das cargas dos átomos de nitrogênio
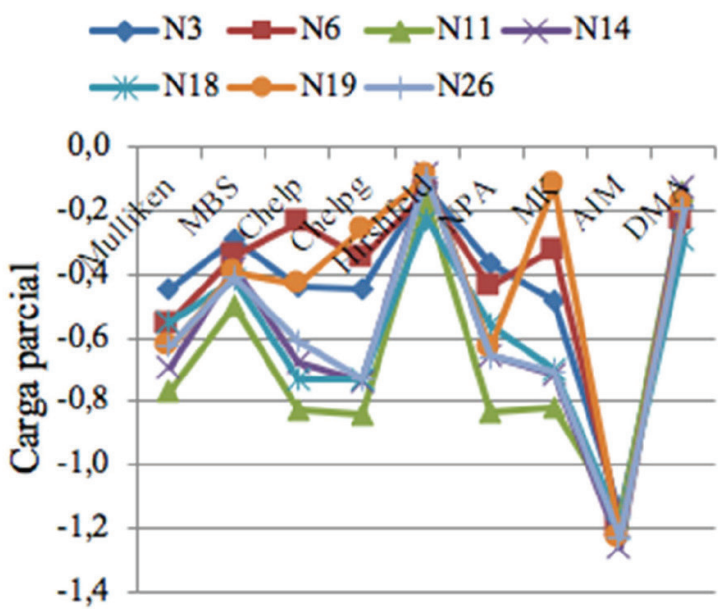

Figura 4. Cargas atômicas calculadas pelos nove diferentes métodos em unidades de e. (a) Variação das cargas dos átomos de oxigênio da molécula; (b) Variação das cargas dos átomos de nitrogênio da molécula

apresentem valores de cargas parciais semelhantes. Isso não ocorreu apenas para o método Chelp, que apresentou uma variação de $20 \%$ entre as cargas desses dois átomos (a segunda maior variação, de $9 \%$, foi devida ao método Chelpg). Assim, vê-se que esses dois métodos, que usam o potencial eletrostático ao redor da molécula para determinar as cargas atômicas, não se mostraram acurados para determinar as cargas dos átomos de oxigênio em questão. Mesmo assim, vale ressaltar que o melhor resultado do método Chelpg em relação ao método Chelp mostra que a forma de distribuição de pontos que formam o grid usado no método Chelpg é de fato uma evolução em relação ao tipo de grid usado no método Chelp. Ainda considerando-se a família de métodos baseados no potencial eletrostático, o método MK se mostrou ainda melhor que os dois primeiros: a diferença entre as cargas MK dos átomos $\mathrm{O} 9$ e $\mathrm{O} 25$ foi de aproximadamente 2,3\%.

Fazendo-se uma comparação análoga para os átomos de nitrogênio N19 e N26, espera-se também que ambos tenham cargas parciais parecidas. Todos os métodos, exceto o de Mulliken, concordaram que o átomo N26 é mais negativo que N19, provavelmente porque a amida formada pelos átomos N19/C8/O9 está próxima o suficiente do anel de pterina da molécula, podendo participar de efeitos de ressonância desse anel. Por conseguinte, a densidade eletrônica dessa amida estaria mais afastada do átomo N19 e mais próxima do anel de pterina. Para os métodos MBS, Hirshfeld, NPA, AIM e DMA, essa participação na ressonância eletrônica do anel não parece ser muito apreciável porque a diferença entre as cargas de N19 e N26 foi próxima de zero. Já os métodos Chelp, Chelpg e MK sugerem que o átomo N26 é muito mais negativo que N19 (para o método MK, essa diferença foi superior a 500\% !), sendo que não há indícios na molécula que comprovem essa diferença tão grande. Concluise, então, que nenhum dos três métodos baseados em potencial eletrostático é eficaz no cálculo dessas cargas.

Como o conceito de grupo funcional está relacionado a um conjunto de propriedades físicas e químicas de uma molécula, foi também realizada uma análise das cargas dos três grupos amida como um todo, somando-se as cargas dos átomos de carbono, nitrogênio e oxigênio formadores de cada grupo amida. Os resultados estão na Tabela 1. A Amida 1 é formada pelos átomos C8, O9 e N19; a Amida 2 formada pelos C16, O17 e N14 e a Amida 3 formada por C24, O25 e N26. Dado que as Amidas 1 e 3 estão em ambientes químicos semelhantes, é de se esperar que suas cargas também sejam parecidas. Essa previsão não se concretizou para os métodos derivados do potencial eletrostático (Chelp, Chelpg e MK), que previram cargas bastantes distintas para os dois grupos funcionais em questão. A maior variação ocorreu para o método MK, no qual a carga da Amida 1 representa apenas 48\% do valor da carga da Amida 3; para os métodos Chelp e Chelpg esses valores percentuais são de $65 \%$ e $61 \%$, respectivamente. A Amida 2, por sua vez, está inserida em um anel de pterina com expressiva ressonância eletrônica, o que diminui a capacidade desse grupo amida de reter a densidade eletrônica em torno de si. Consequentemente, espera-se que a Amida 2 concentre em torno de si menos densidade eletrônica e, portanto, tenha uma carga menos negativa que as cargas das Amidas 1 e 3. Apenas os métodos MBS, Hirshfeld, NPA e DMA previram cargas concordantes com essa previsão.

Também foram comparadas as cargas parciais dos átomos constituintes dos três grupos amida presentes na molécula de NNPT, cujos valores encontram-se na Tabela 1. Os átomos de carbono destes grupos apresentaram cargas positivas com módulos razoavelmente grandes. Os valores médios de cargas parciais atômicas desses três átomos empregando-se os nove métodos foram: $(0,5486 e \pm 0,3910 e)$ para o átomo $\mathrm{C} 8 ;(0,58746 e \pm 0,3598 e)$ para o átomo $\mathrm{C} 16$ e $(0,6031 e \pm$ 0,3908 e) para o átomo C24. Embora os desvios-padrão dessas cargas estejam altos, indicando grande dispersão dos valores calculados pelos diferentes métodos, esses valores bastante positivos de cargas parciais são esperados dada a ligação química direta desses átomos de carbono com átomos muito mais eletronegativos. Ainda considerando-se o efeito das diferenças de eletronegatividade, o átomo de oxigênio, sendo o mais eletronegativo do grupo amida, deve apresentar a carga mais negativa. Entretanto, apenas os métodos MBS, Hirshfeld e DMA forneceram valores de cargas compatíveis com esta análise para os três grupos amida.

Foram também comparadas as cargas atômicas de pares de átomos de hidrogênio ligados a um mesmo átomo pesado, a saber, H12-H13, H22-H23 e H35-H36 (Figura 5). O eixo $y$ mostra os valores percentuais $(\%)$ da diferença entre as cargas dos átomos de hidrogênio em relação à carga média dos mesmos átomos, conforme Equação 7,

$$
\%=\frac{q_{H x}-q_{H y}}{\left[\left(q_{H x}+q_{H y}\right) / 2\right]} \times 100
$$


em que $q_{H i}$ é o valor da carga do átomo $i$ e $x<y$.

Considerando-se o par de átomos de hidrogênio H12-H13, observa-se que todos os métodos apontaram uma diferença percentual positiva entre as cargas desses dois átomos. Dado que as cargas de ambos os átomos são positivas segundo todos os métodos de cálculo considerados, conclui-se que a carga de $\mathrm{H} 12$ deve ser maior que a de H13. Pelas Figuras 2 e 6, nota-se que a geometria do átomo N11 é trigonal planar, indicando a participação do par de elétrons livres desse átomo em efeitos de conjugação eletrônica com os átomos C10 e N14 e, portanto, limitando a rotação da ligação C10-N11 já que o alinhamento de orbitais $p$ é necessário para ocorrer essa conjugação eletrônica. Assim, os átomos de hidrogênio H12 e H13 têm posições relativamente fixas, estando o átomo $\mathrm{H} 12$ mais próximo de $\mathrm{N} 18$, que tem hibridização $\mathrm{sp}^{2}$, e o átomo $\mathrm{H} 13$ mais próximo de N14, que tem hibridização $\mathrm{sp}^{3}$. Assim, conforme esperado, devido a esta diferença de eletronegatividade, a média das cargas calculadas por todos os métodos é -0,507e para o átomo N14 e -0,526e para N18. Essa assimetria de distribuição de cargas em átomos próximos aos átomos H12 e H13 parece ser a causa da diferença de cargas entre esses dois átomos de hidrogênio, sendo que a maior concentração de carga negativa próxima ao N18 causou a maior deficiência de elétrons em H12, que está mais próximo do átomo N18. Apesar disso, a diferença percentual máxima foi de $12,8 \%$ para o método DMA, indicando que a diferença de cargas entre esses dois átomos de hidrogênio não é muito expressiva.

Os átomos H22 e H23 estão ligados ao átomo C21, que tem hibridização $\mathrm{sp}^{3}$ e está situado na região central da molécula. Dado que as ligações simples C21-N19 e C21-C24 podem girar livremente, é de se esperar que as posições dos átomos H22 e H23 sejam razoavelmente equivalentes. As pequenas diferenças percentuais observadas na Figura 5 para todos os métodos exceto o Chelp corroboram essa interpretação. O método Chelp, além de apresentar cargas com diferença percentual muito superior às dos outros métodos, ainda produziu cargas negativas nos átomos $\mathrm{H} 22 \mathrm{e}$ H23, o que é quimicamente impossível já que esses átomos são menos eletronegativos que o átomo de carbono a que estão ligados. Logo, o método Chelp se mostrou ineficaz no cálculo das cargas desses dois átomos de hidrogênio.

Tabela 1. Valores de cargas parciais dos átomos que constituem os três grupos amida da molécula

\begin{tabular}{|c|c|c|c|c|c|c|c|c|c|}
\hline Átomo & Mulliken & MBS & Chelp & Chelpg & Hirshfeld & NPA & MK & AIM & DMA \\
\hline C 8 & 0,5916 & 0,4201 & 0,5562 & 0,4428 & 0,1645 & 0,6541 & 0,3915 & 1,4980 & 0,2185 \\
\hline O 9 & $-0,5387$ & $-0,5043$ & $-0,5176$ & $-0,5090$ & $-0,3144$ & $-0,6285$ & $-0,4892$ & $-1,1876$ & $-0,4332$ \\
\hline N 19 & $-0,6294$ & $-0,3958$ & $-0,4286$ & $-0,2587$ & $-0,0917$ & $-0,6349$ & $-0,1154$ & $-1,2327$ & $-0,1741$ \\
\hline Amida 1 & $-0,5765$ & $-0,4801$ & $-0,3899$ & $-0,3248$ & $-0,2417$ & $-0,6093$ & $-0,2132$ & $-0,9223$ & $-0,3888$ \\
\hline C 16 & 0,6051 & 0,4263 & 0,6092 & 0,6339 & 0,1712 & 0,6642 & 0,6000 & 1,4280 & 0,2386 \\
\hline O 17 & $-0,4728$ & $-0,4366$ & $-0,5248$ & $-0,4998$ & $-0,2881$ & $-0,5629$ & $-0,4856$ & $-1,1475$ & $-0,3494$ \\
\hline N 14 & $-0,6903$ & $-0,3805$ & $-0,6794$ & $-0,7390$ & $-0,0789$ & $-0,6538$ & $-0,7132$ & $-1,2596$ & $-0,1244$ \\
\hline Amida 2 & $-0,5580$ & $-0,3908$ & $-0,5951$ & $-0,6049$ & $-0,1957$ & $-0,5525$ & $-0,5989$ & $-0,9790$ & $-0,2352$ \\
\hline C 24 & 0,6056 & 0,4297 & 0,4271 & 0,6622 & 0,1810 & 0,6906 & 0,7386 & 1,4925 & 0,2008 \\
\hline O 25 & $-0,5159$ & $-0,4856$ & $-0,4140$ & $-0,4632$ & $-0,2933$ & $-0,6289$ & $-0,4778$ & $-1,1887$ & $-0,4288$ \\
\hline N 26 & $-0,6272$ & $-0,4081$ & $-0,6124$ & $-0,7308$ & $-0,0940$ & $-0,6578$ & $-0,7053$ & $-1,2331$ & $-0,1976$ \\
\hline Amida 3 & $-0,5375$ & $-0,4640$ & $-0,5993$ & $-0,5318$ & $-0,2063$ & $-0,5961$ & $-0,4445$ & $-0,9293$ & $-0,4256$ \\
\hline
\end{tabular}

Variação percentual entre cargas de hidrogênios ligados a um mesmo átomo pesado

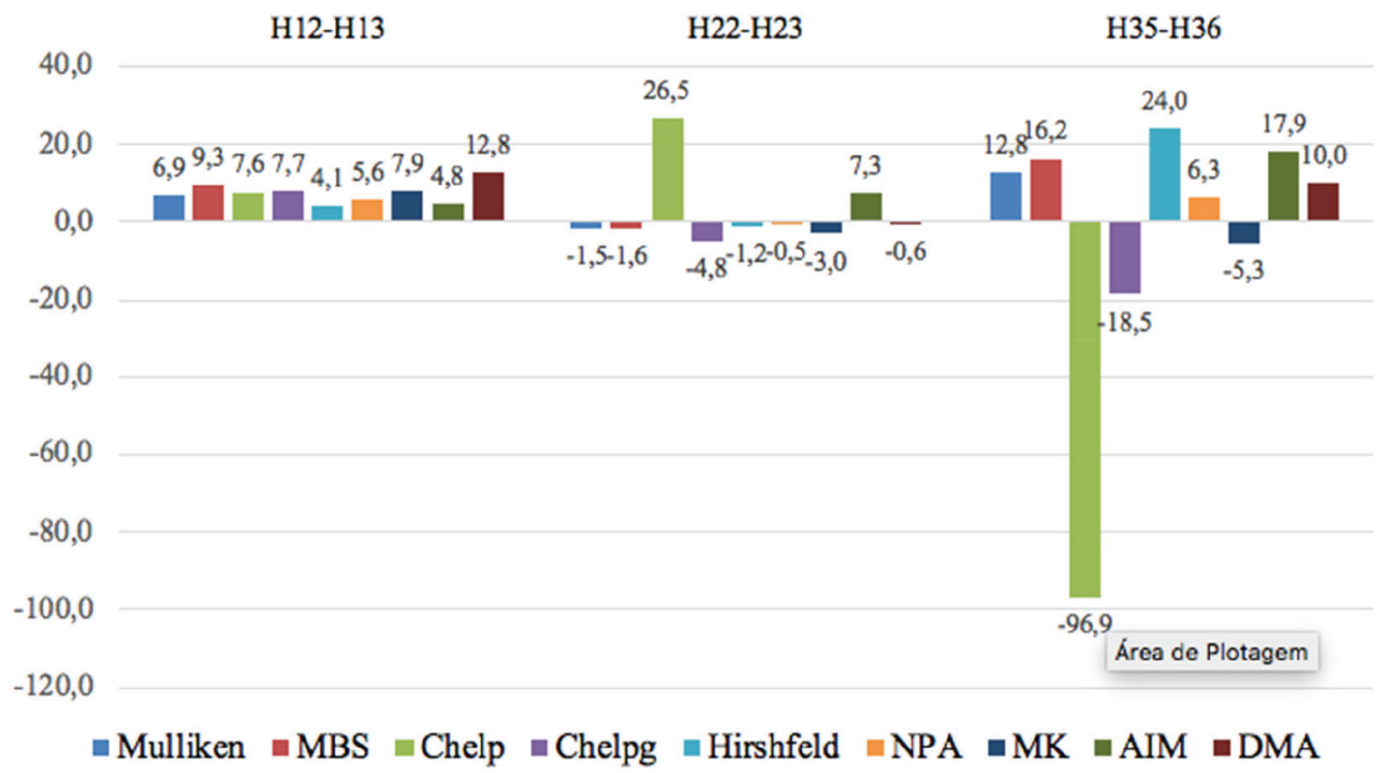

Figura 5. Comparação das cargas de átomos de hidrogênio selecionados 


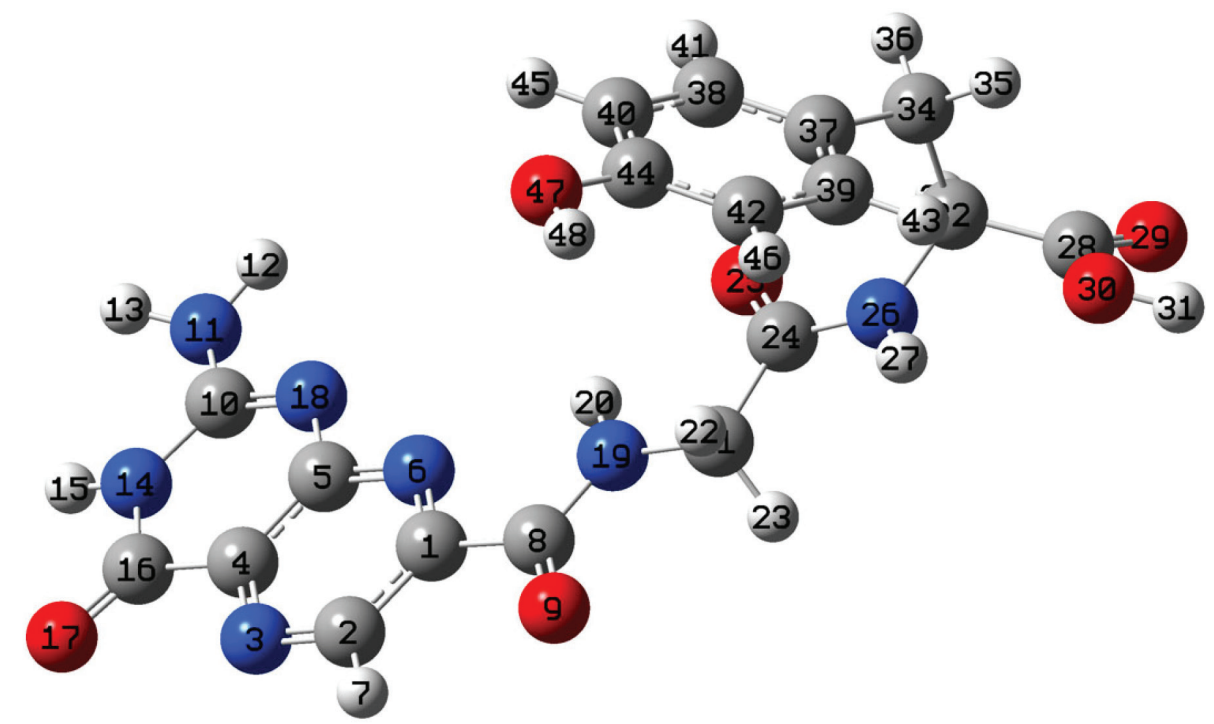

Figura 6. Molécula de NNPT com rotação de $180^{\circ}$ da ligação C34 - C32

Finalmente, comparando-se as cargas dos átomos H35 e H36 percebe-se esse par de átomos de hidrogênio foi o que apresentou, de maneira geral, as maiores diferenças percentuais para cada método de cálculo de carga. Isso era esperado pois os átomos H35 e H36 estão em ambientes químicos distintos.

Analisando-se mais uma vez a molécula de NNPT na Figura 2, vemos que, em princípio, as ligações sigma C34-C37 e C34-C32 teriam rotação livre. Entretanto, rotações da ligação C34-C32 aproximariam o anel benzênico de outras regiões ricas em elétrons da molécula, o que geraria repulsão e, consequentemente, instabilidade. Isso pode ser visto na Figura 6, onde a molécula de NNPT teve a ligação C34-C32 girada em $180^{\circ}$ e a proximidade entre os átomos fica clara. Essas restrições de rotação fazem com que as posições dos átomos H35 e H36 não sejam totalmente equivalentes e, portanto, espera-se uma diferença entre os valores das suas cargas. Mais uma vez, o método Chelp atribuiu cargas negativas a esses dois átomos e ainda apresentou uma diferença percentual de quase $-97 \%$ entre essas cargas, mostrando-se um método inadequado para cálculo de cargas de átomos em moléculas similares à NNPT. Além disso, não houve diferença entre os métodos no que concerne o sinal da diferença das cargas dos hidrogênios 35 e 36. Como o átomo H36 está mais próximo da carboxila formada por C28-O29-O30-H31 e esse grupo concentra densidade eletrônica em torno de si devido à alta eletronegatividade dos átomos de oxigênio, é esperado que $\mathrm{H} 36$ seja mais positivo que H35, portanto os métodos Chelpg e MK previram corretamente que H35 tem carga menos positiva que H36.

\section{Análise do vetor momento de dipolo elétrico calculado pelos diferentes métodos}

Utilizando-se cada um dos nove métodos de cálculo de cargas atômicas, também foi determinado o momento de dipolo elétrico total da molécula de NNPT. As coordenadas $x, y, z$ (em $\AA$ ) e os módulos do vetor momento de dipolo (em Debye) estão listadas na Tabela 2. A Figura 7 mostra esses vetores posicionados relativamente à molécula.

De maneira geral, observa-se que o módulo, direção e o sentido dos vetores determinados pelos diferentes métodos são bastante similares. Considerando-se todos os nove métodos, o valor médio do módulo do vetor momento de dipolo foi de $(6,226 \pm 0,165) \mathrm{D}$. O baixo valor do desvio padrão em relação ao valor do módulo do vetor confirma que houve uma boa concordância quanto ao módulo do vetor momento de dipolo. O sentido dos vetores indica que o anel benzênico é a região mais positiva da molécula, o que está consistente com o esperado do ponto de vista químico. Átomos mais eletronegativos concentram em torno de si densidade eletrônica, então a região esquerda da molécula de NNPT, conforme representada na Figura 2, está deficiente em elétrons e, portanto, é a área mais positiva da molécula. Pelos dados da Tabela 2, vê-se que os valores da coordenada $x$ calculados pelos métodos Chelp e AIM são ligeiramente mais negativos que os calculados pelos outros sete métodos. Destacase, ainda, que os três métodos derivados do potencial eletrostático (Chelp, Chelpg e MK) apresentaram um valor mais negativo para a coordenada $z$ do vetor, de acordo com a Tabela 2, o que pode ter ocorrido por consequência da forma de cálculo de cargas parciais atômicas desses três métodos.

\section{Análise da variação das cargas atômicas de acordo com variações de tamanho de base e método de cálculo}

De modo a se verificar o comportamento dos métodos de cálculo de carga com variações de tamanho de base e de método quântico ou DFT, as cargas dos átomos da NNPT foram calculadas pelos métodos DFT/B3LYP e MP2 ${ }^{61}$ com conjunto de base 6-311++G(d,p). Os valores de cargas atômicas obtidos encontram-se nas Tabelas 3S e 4S do Material Suplementar, respectivamente. A Tabela 3 mostra os valores de desvio padrão de cargas calculadas para um

Tabela 2. Coordenadas dos vetores momento de dipolo calculados pelos nove métodos de cálculo de carga atômica. Unidades: Debye (D)

\begin{tabular}{cccccccccc}
\hline & Mulliken & MBS & Chelp & Chelpg & Hirshfeld & NPA & MK & AIM & DMA \\
\hline x $(\AA)$ & $-2,8571$ & $-2,8571$ & $-3,1416$ & $-2,8861$ & $-2,8512$ & $-2,8570$ & $-2,9336$ & $-3,2064$ & $-2,8580$ \\
y $(\AA)$ & 5,3954 & 5,3955 & 5,3763 & 5,4655 & 5,3936 & 5,3955 & 5,4469 & 5,4340 & 5,3953 \\
z (̊) & $-0,4582$ & $-0,4582$ & $-0,6578$ & $-0,6777$ & $-0,4576$ & $-0,4600$ & $-0,5665$ & $-0,5304$ & $-0,4580$ \\
Módulo (D) & 6,1223 & 6,1225 & 6,2615 & 6,2177 & 6,1180 & 6,1225 & 6,6126 & 6,3317 & 6,1227 \\
\hline
\end{tabular}




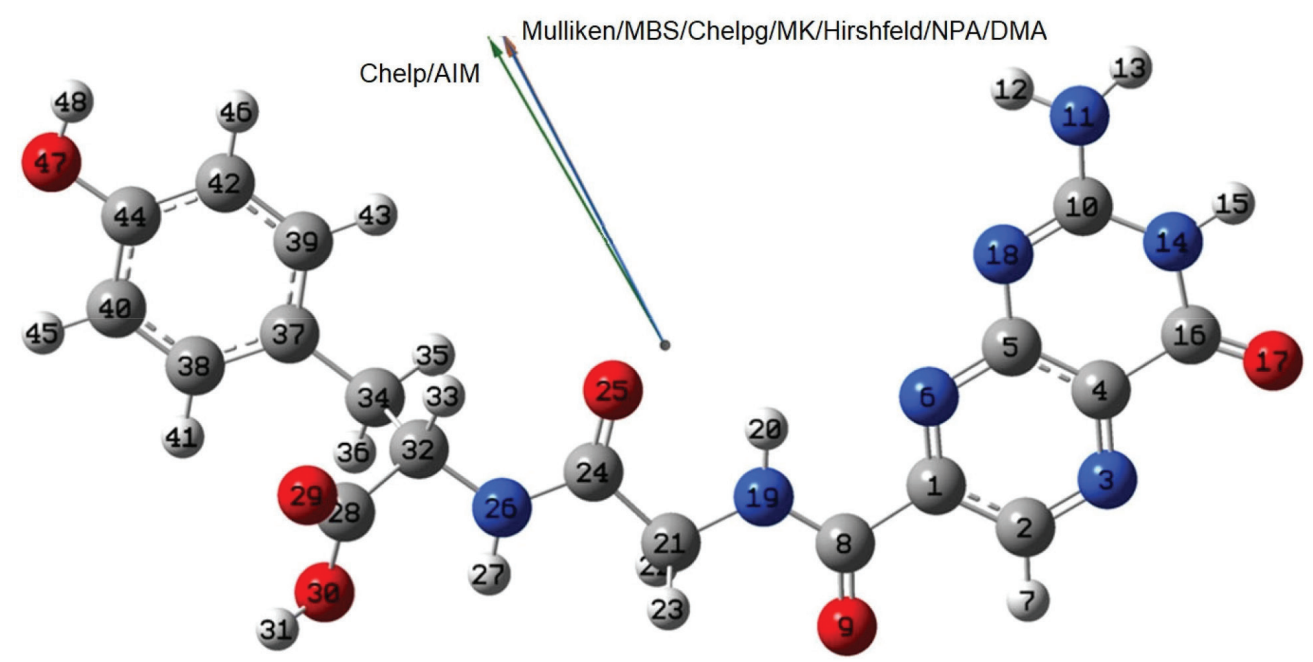

Figura 7. Vetor momento de dipolo elétrico determinados pelos nove métodos de cálculo de carga atômica. A origem dos vetores coincide com o centro de massa da molécula

mesmo átomo por um mesmo método de cálculo de cargas e os três diferentes métodos quânticos e tamanho de conjunto de base. Os valores da Tabela 3 foram coloridos segundo uma escala de cores em que valores menores estão em tons de verde e valores maiores, em tons de vermelho.

Conforme o previsto, o método de Mulliken é bastante sensível a variações de conjunto de base e de método de cálculo, sendo o que apresentou maiores valores de desvio padrão, representados pelas células em tons de vermelho. As cargas calculadas pelo método MBS já são menos suscetíveis a estas mudanças, conforme previsto pela teoria do método. Os métodos Hirshfeld e DMA são os que contêm mais células em tons de verde na Tabela 3, mostrando maior independência do método de cálculo e do conjunto de base.

A análise das Tabelas $2 \mathrm{~S}, 3 \mathrm{~S}$ e $4 \mathrm{~S}$ mostra, ainda, que muitas tendências dos métodos de cálculo de carga se mantêm ao se variar o nível de teoria utilizado para esse cálculo. As cargas de Hirshfeld continuam sendo muitas vezes as menores em módulo, enquanto as cargas AIM de átomos eletronegativos têm valores muito mais negativos que as calculadas por outros métodos, o que acarreta também a atribuição de cargas muito positivas àqueles ligados diretamente aos átomos mais eletronegativos. Adicionalmente, o método Chelpg atribuiu erroneamente cargas negativas a alguns átomos de hidrogênio em todos os níveis de teoria. Os métodos MBS e DMA, que já haviam concordado com as previsões de tendências de cargas atômicas em nível DFT/B3LYP/G-31G(d), foram ainda os menos afetados, juntamente com o método de Hirshfeld, às variações de níveis de teoria, indicando a adequação desses métodos para determinar cargas parciais de sistemas moleculares similares à NNPT segundo todos os níveis de teoria contemplados neste trabalho.

\section{CONCLUSÕES}

Neste trabalho, as cargas parciais atômicas da molécula de NNPT foram determinados por nove diferentes métodos, a saber: Mulliken, MBS Mulliken, Chelp, Chelpg, MK, Hirshfeld, NPA, AIM e DMA. Os métodos de cálculo de cargas atômicas utilizam diferentes abordagens para determinar esses valores e nota-se que a maneira como esses métodos descrevem efeitos indutivos, de deslocalização eletrônica e de diferenças de eletronegatividade leva a diferenças expressivas nos valores das cargas atribuídas a um mesmo átomo.

Para a molécula estudada, os métodos derivados do potencial eletrostático (Chelp, Chelpg e MK) foram considerados os menos apropriados para os átomos analisados por apresentarem a maior quantidade de resultados diferentes dos esperados. Cargas AIM tendem a ter módulos significativamente maiores quando comparadas com as calculadas pelos outros métodos, conforme resultados prévios já reportados. ${ }^{6,40}$

Os métodos considerados mais precisos do ponto de vista químico, que na maior parte dos casos forneceram valores de cargas parciais quimicamente coerentes e bastante insensíveis a variações de níveis de teoria foram: MBS, DMA e Hirshfeld. Entretanto, a tendência do método Hirshfeld de atribuir cargas atômicas muito próximas de zero se confirmou no estudo realizado, portanto, as cargas atômicas calculadas por este método devem ser analisadas com cuidado antes de serem usadas para interpretar fenômenos químicos.

Por conseguinte, os métodos MBS e DMA foram considerados os mais apropriados para cálculo de cargas atômicas e vetor momento de dipolo elétrico da molécula de NNPT e possivelmente para sistemas moleculares semelhantes, em todos os níveis de teoria abordados neste trabalho. Por essa razão, são os dois métodos recomendados em trabalhos de dinâmica molecular e em outros problemas que necessitem de cargas atômicas precisas do ponto de vista químico. As referidas cargas podem inclusive ser utilizadas em reparametrizações de campos de força que necessitam de cargas atômicas, atuando como complementos ou mesmo substituições de cálculos de carga pelo método RESP, usualmente utilizado, quando esse último não for capaz de reproduzir resultados experimentais.

\section{MATERIAL SUPLEMENTAR}

Estão contidas no Material Suplementar as Tabelas 1S, 2S, 3S e 4S. A Tabela $1 \mathrm{~S}$ contém os valores de comprimentos de ligação calculados pelo método DFT/B3LYP; as Tabelas 2S, 3S e 4S contêm os valores de cargas parciais de todos os átomos da molécula de NNPT calculados pelos nove métodos considerados neste trabalho segundo os níveis de teoria DFT/B3LYP/G-31G(d), DFT/B3LYP/G$311++\mathrm{G}(\mathrm{d}, \mathrm{p})$ e $\mathrm{MP} 2 / \mathrm{G}-311++\mathrm{G}(\mathrm{d}, \mathrm{p})$, respectivamente.

\section{AGRADECIMENTOS}

I. B. J. agradece o apoio do Conselho Nacional de Pesquisa (CNPq) (304148/2018-0 e 409447/2018-8) e do Exército Brasileiro a essa pesquisa. T. C. C. F. agradece o apoio do CNPq (308225/2018-0) e da Fundação de Amparo a Pesquisa do Estado do Rio de Janeiro (FAPERJ) (E-02/202.961/2017). Esse trabalho teve também o suporte da Universidade de Hradec Králové. 
Tabela 3. Desvio padrão dos resultados de cargas atômicas (em unidades de $e$ ) entre os métodos DFT/B3LYP com conjunto de base G-31G(d), DFT/B3LYP com conjunto de base 6-311++G(d,p) e MP2 com conjunto de base 6-311++G(d,p)

\begin{tabular}{|c|c|c|c|c|c|c|c|c|c|}
\hline Átomo & Mulliken & MBS & Chelp & Chelpg & Hirshfeld & NPA & MK & AIM & DMA \\
\hline $\mathrm{C} 1$ & 0,054 & 0,013 & 0,038 & 0,008 & 0,012 & 0,020 & 0,005 & 0,026 & 0,014 \\
\hline $\mathrm{C} 2$ & 0,058 & 0,007 & 0,050 & 0,029 & 0,003 & 0,018 & 0,028 & 0,019 & 0,008 \\
\hline N3 & 0,250 & 0,005 & 0,014 & 0,016 & 0,002 & 0,007 & 0,017 & 0,063 & 0,003 \\
\hline $\mathrm{C} 4$ & 0,312 & 0,012 & 0,043 & 0,034 & 0,003 & 0,025 & 0,027 & 0,035 & 0,006 \\
\hline $\mathrm{C} 5$ & 0,512 & 0,038 & 0,065 & 0,041 & 0,016 & 0,049 & 0,037 & 0,058 & 0,022 \\
\hline N6 & 0,335 & 0,024 & 0,048 & 0,011 & 0,011 & 0,027 & 0,015 & 0,053 & 0,015 \\
\hline $\mathrm{H} 7$ & 0,073 & 0,007 & 0,027 & 0,016 & 0,002 & 0,020 & 0,022 & 0,007 & 0,002 \\
\hline $\mathrm{C} 8$ & 0,417 & 0,076 & 0,089 & 0,132 & 0,038 & 0,086 & 0,115 & 0,069 & 0,046 \\
\hline O9 & 0,096 & 0,049 & 0,049 & 0,067 & 0,032 & 0,053 & 0,063 & 0,034 & 0,035 \\
\hline $\mathrm{C} 10$ & 0,228 & 0,076 & 0,090 & 0,100 & 0,034 & 0,093 & 0,077 & 0,082 & 0,045 \\
\hline N11 & 0,257 & 0,040 & 0,033 & 0,062 & 0,010 & 0,030 & 0,057 & 0,037 & 0,012 \\
\hline H12 & 0,032 & 0,012 & 0,011 & 0,018 & 0,004 & 0,013 & 0,018 & 0,006 & 0,004 \\
\hline H13 & 0,047 & 0,011 & 0,014 & 0,019 & 0,004 & 0,014 & 0,018 & 0,011 & 0,006 \\
\hline N14 & 0,170 & 0,055 & 0,081 & 0,076 & 0,016 & 0,048 & 0,051 & 0,069 & 0,021 \\
\hline H15 & 0,036 & 0,013 & 0,019 & 0,022 & 0,004 & 0,014 & 0,017 & 0,013 & 0,003 \\
\hline $\mathrm{C} 16$ & 0,192 & 0,076 & 0,116 & 0,108 & 0,037 & 0,088 & 0,086 & 0,057 & 0,045 \\
\hline O17 & 0,090 & 0,048 & 0,064 & 0,063 & 0,031 & 0,053 & 0,057 & 0,028 & 0,035 \\
\hline N18 & 0,178 & 0,053 & 0,068 & 0,066 & 0,028 & 0,069 & 0,058 & 0,054 & 0,036 \\
\hline N19 & 0,217 & 0,055 & 0,062 & 0,113 & 0,017 & 0,047 & 0,088 & 0,056 & 0,022 \\
\hline $\mathrm{H} 20$ & 0,042 & 0,017 & 0,048 & 0,027 & 0,006 & 0,007 & 0,025 & 0,006 & 0,004 \\
\hline $\mathrm{C} 21$ & 0,112 & 0,033 & 0,035 & 0,056 & 0,014 & 0,076 & 0,030 & 0,017 & 0,021 \\
\hline $\mathrm{H} 22$ & 0,024 & 0,009 & 0,016 & 0,006 & 0,002 & 0,029 & 0,005 & 0,011 & 0,005 \\
\hline $\mathrm{H} 23$ & 0,028 & 0,009 & 0,011 & 0,006 & 0,002 & 0,029 & 0,006 & 0,006 & 0,004 \\
\hline $\mathrm{C} 24$ & 0,309 & 0,076 & 0,094 & 0,092 & 0,037 & 0,087 & 0,089 & 0,072 & 0,045 \\
\hline $\mathrm{O} 25$ & 0,100 & 0,053 & 0,078 & 0,057 & 0,035 & 0,056 & 0,058 & 0,034 & 0,036 \\
\hline N26 & 0,457 & 0,055 & 0,060 & 0,096 & 0,017 & 0,045 & 0,109 & 0,060 & 0,023 \\
\hline $\mathrm{H} 27$ & 0,026 & 0,011 & 0,013 & 0,015 & 0,004 & 0,014 & 0,017 & 0,017 & 0,005 \\
\hline $\mathrm{C} 28$ & 0,542 & 0,076 & 0,049 & 0,093 & 0,039 & 0,080 & 0,076 & 0,057 & 0,046 \\
\hline O29 & 0,141 & 0,049 & 0,051 & 0,066 & 0,031 & 0,053 & 0,059 & 0,037 & 0,035 \\
\hline $\mathrm{O} 30$ & 0,240 & 0,050 & 0,050 & 0,054 & 0,023 & 0,033 & 0,056 & 0,025 & 0,020 \\
\hline H31 & 0,063 & 0,018 & 0,039 & 0,019 & 0,007 & 0,007 & 0,022 & 0,006 & 0,006 \\
\hline C32 & 0,476 & 0,024 & 0,263 & 0,071 & 0,012 & 0,047 & 0,109 & 0,016 & 0,015 \\
\hline H33 & 0,060 & 0,007 & 0,050 & 0,013 & 0,002 & 0,032 & 0,021 & 0,009 & 0,004 \\
\hline C34 & 0,031 & 0,022 & 0,136 & 0,015 & 0,008 & 0,066 & 0,016 & 0,021 & 0,015 \\
\hline H35 & 0,032 & 0,009 & 0,013 & 0,005 & 0,004 & 0,031 & 0,003 & 0,008 & 0,006 \\
\hline H36 & 0,050 & 0,010 & 0,022 & 0,008 & 0,003 & 0,030 & 0,004 & 0,012 & 0,007 \\
\hline C37 & 0,546 & 0,009 & 0,041 & 0,012 & 0,002 & 0,011 & 0,006 & 0,011 & 0,004 \\
\hline C38 & 0,299 & 0,013 & 0,051 & 0,007 & 0,007 & 0,034 & 0,009 & 0,014 & 0,011 \\
\hline C39 & 0,292 & 0,012 & 0,029 & 0,004 & 0,008 & 0,033 & 0,008 & 0,021 & 0,011 \\
\hline $\mathrm{C} 40$ & 0,058 & 0,005 & 0,110 & 0,039 & 0,002 & 0,018 & 0,051 & 0,015 & 0,005 \\
\hline H41 & 0,040 & 0,007 & 0,006 & 0,009 & 0,002 & 0,023 & 0,016 & 0,011 & 0,006 \\
\hline $\mathrm{C} 42$ & 0,041 & 0,005 & 0,064 & 0,031 & 0,003 & 0,018 & 0,065 & 0,014 & 0,005 \\
\hline H43 & 0,054 & 0,007 & 0,023 & 0,015 & 0,002 & 0,023 & 0,019 & 0,013 & 0,004 \\
\hline $\mathrm{C} 44$ & 0,493 & 0,029 & 0,106 & 0,060 & 0,015 & 0,032 & 0,084 & 0,021 & 0,017 \\
\hline H45 & 0,051 & 0,007 & 0,032 & 0,018 & 0,003 & 0,020 & 0,023 & 0,013 & 0,005 \\
\hline $\mathrm{H} 46$ & 0,035 & 0,007 & 0,029 & 0,012 & 0,003 & 0,020 & 0,025 & 0,014 & 0,006 \\
\hline O47 & 0,223 & 0,045 & 0,040 & 0,054 & 0,023 & 0,028 & 0,056 & 0,022 & 0,022 \\
\hline $\mathrm{H} 48$ & 0,080 & 0,016 & 0,008 & 0,016 & 0,007 & 0,010 & 0,016 & 0,008 & 0,007 \\
\hline
\end{tabular}




\section{REFERÊNCIAS}

1. Kelly, C. P.; Cramer, C. J.; Truhlar, D. G.; Theor. Chem. Acc. 2005, 113, 133.

2. Thompson, J. D.; Xidos, J. D.; Sonbuchner, T. M.; Cramer, C. J.; Truhlar, D. G.; PhysChemComm 2002, 5, 117.

3. Foresman, J. B.; Frisch, Æ.; Exploring Chemistry with Electronic Structure Methods, $2^{\text {nd }}$ ed., Gaussian, Inc.: Pittsburgh, 1995.

4. Leach, A. R.; Molecular Modelling: Principles and Applications, $2^{\text {nd }}$ ed., Prentice Hall: Harlow, 2001.

5. Wang, B.; Li, S. L.; Truhlar, D. G.; J. Chem. Theory Comput. 2014, 10 , 5640 .

6. Matczak, P.; Computation (2016), doi:10.3390/computation4010003.

7. Heinz, H.; Suter, U. W.; J. Phys. Chem. B 2004, 108, 18341.

8. Pagadala, N. S.; Syed, K.; Tuszynski, J.; Biophys. Rev. 2017, 9, 91.

9. Durrant, J. D.; McCammon, J. A.; BMC Biol. 2011, 9, 71.

10. Gioia, D.; Bertazzo, M.; Recanatini, M.; Masetti, M.; Cavalli, A.; Molecules 2017, 22, 1

11. Mulliken, R. S.; J. Chem. Phys. 1955, 23, 1833.

12. Montgomery, J. A.; Frisch, M. J.; Ochterski, J. W.; Petersson, G. A.; J. Chem. Phys. 2000, 112, 6532.

13. Reed, A. E.; Weinstock, R. B.; Weinhold, F.; J. Chem. Phys. 1985, 83, 735 .

14. Stone, A. J.; Alderton, M.; Mol. Phys. 1985, 56, 1047.

15. Bader, R. F. W.; Acc. Chem. Res. 1985, 18, 9.

16. Hirshfeld, F. L.; Theor. Chim. Acta 1977, 44, 129.

17. Chirlian, L. E.; Francl, M. M.; J. Comput. Chem. 1987, 8, 894.

18. Breneman, C. M.; Wiberg, K. B.; J. Comput. Chem. 1990, 11, 361.

19. Singh, U. C.; Kollman, P. A.; J. Comput. Chem. 1984, 5, 129.

20. Witter, R.; Möllhoff, M.; Koch, F. T.; Sternberg, U.; J. Chem. (2015), doi: $10.1155 / 2015 / 908204$

21. Guadagnini, P. H.; Bruns, R. E.; Quim. Nova 1996, 19, 148.

22. Cramer, C. J. Essentials of Computational Chemistry - Theories and Models, $2^{\text {nd }}$ ed., John Wiley \& Sons: Hoboken, 2004.

23. Voityuk, A. A.; Stasyuk, A. J.; Vyboishchikov, S. F.; Phys. Chem. Chem. Phys. 2018, 20, 23328.

24. Dupradeau, F. Y.; Cézard, C.; Lelong, R.; Stanislawiak, É.; Pêcher, J.; Delepine, J. C.; Cieplak, P.; Nucleic Acids Res. 2008, 36, 360.

25. Vanommeslaeghe, K.; Raman, E. P.; MacKerell, A. D.; J. Chem. Inf. Model. 2012, 52, 3155.

26. Halgren, T. A.; J. Comput. Chem. 1996, 17, 520.

27. Jorgensen, W. L.; Tirado-Rives, J.; J. Am. Chem. Soc. 1988, 110, 1657.

28. Jorgensen, W. L.; Maxwell, D. S.; Tirado-Rives, J.; J. Am. Chem. Soc. 1996, 118,11225 .

29. Kaminski, G. A.; Friesner, R. A.; Tirado-Rives, J.; Jorgensen, W. L.; J. Phys. Chem. B 2001, 105, 6474.

30. Cornell, W. D.; Cieplak, P.; Bayly, C. I.; Gould, I. R.; Merz, K. M.; Ferguson, D. M.; Spellmeyer, D. C.; Fox, T.; Caldwell, J. W.; Kollman, P. A.; J. Am. Chem. Soc. 1995, 117, 5179

31. Duan, Y.; Wu, C.; Chowdhury, S.; Lee, M. C.; Xiong, G.; Zhang, W.; Yang, R.; Cieplak, P.; Luo, R.; Lee, T.; Caldwell, J.; Wang, J.; Kollman, P.; J. Comput. Chem. 2003, 24, 1999.

32. Bayly, C. I.; Cieplak, P.; Cornell, W. D.; Kollman, P. A.; J. Phys. Chem. 1993, 97, 10269

33. Hu, Z.; Jiang, J.; J. Comput. Chem. 2009, 31, 371.
34. Mecklenfeld, A.; Raabe, G.; J. Chem. Theory Comput. 2017, 13, 6266.

35. Saito, R.; Pruet, J. M.; Manzano, L. A.; Jasheway, K.; Monzingo, A. F.; Wiget, P. A.; Kamat, I.; Anslyn, E. V.; Robertus, J. D.; J. Med. Chem. 2013, 56, 320.

36. Balint, G. A.; Toxicology 1974, $2,77$.

37. Anders, G.; Borges, I.; J. Phys. Chem. A 2011, 115, 9055.

38. Wiberg, K. B.; Rablen, P. R.; J. Org. Chem. 2018, 83, 15463.

39. Bader, R. F. W.; Atoms in Molecules - a Quantum Theory, $1^{\text {st }}$ ed., Wiley: Chichester, 1990.

40. Fogarty, R. M.; Matthews, R. P.; Ashworth, C. R.; Brandt-Talbot, A.; Palgrave, R. G.; Bourne, R. A.; Vander Hoogerstraete, T.; Hunt, P. A.; Lovelock, K. R. J.; J. Chem. Phys. 2018, 148, 193817.

41. Bultinck, P.; Van Alsenoy, C.; Ayers, P. W.; Carbó-Dorca, R.; J. Chem. Phys. 2007, 126, 144111.

42. Ayers, P. W.; J. Chem. Phys. 2000, 113, 10886.

43. Nikolova, V.; Cheshmedzhieva, Di.; Ilieva, S.; Galabov, B.; J. Org. Chem. 2019, 84, 1908.

44. Liu, S.; J. Chem. Phys. 2014, 141, 194109.

45. Price, S. L.; Stone, A. J.; Chem. Phys. Lett. 1983, 98, 419.

46. Borges Jr, I.; Int. J. Quantum Chem. 2008, 108, 2615.

47. Oliveira, M. A. S.; Borges, I.; Int. J. Quantum Chem. 2019, 119, 1.

48. de Oliveira, R. S. S.; Borges, I.; J. Mol. Model. 2019, 25, 314.

49. Silva, A. M.; Borges, I.; J. Comput. Chem. 2011, 32, 2186.

50. Borges, I.; Silva, A. M.; Aguiar, A. P.; Borges, L. E. P.; Santos, J. C. A.; Dias, M. H. C.; J. Mol. Struct.: THEOCHEM 2007, 822, 80.

51. Borges, I.; Silva, A. M.; J. Braz. Chem. Soc. 2012, 23, 1789.

52. Frisch, M. J.; Trucks, G. W.; Schlegel, H. B.; Scuseria, G. E.; Robb, M. A.; Cheeseman, J. R.; Scalmani, G.; Barone, V.; Mennucci, B.; Petersson, G. A.; Nakatsuji, H.; Caricato, M.; Li, X.; Hratchian, H. P.; Izmaylov, A. F.; Bloino, J.; Zheng, G.; Sonnenberg, J. L.; Hada, M.; Ehara, M.; Toyota, K.; Fukuda, R.; Hasegawa, J.; Ishida, M.; Nakajima, T.; Honda, Y.; Kitao, O.; Nakai, H.; Vreven, T.; Montgomery, Jr., J. A.; Peralta, J. E.; Ogliaro, F.; Bearpark, M.; Heyd, J. J.; Brothers, E.; Kudin, K. N.; Staroverov, V. N.; Kobayashi, R.; Normand, J.; Raghavachari, K.; Rendell, A.; Burant, J. C.; Iyengar, S. S.; Tomasi, J.; Coss, M.; Rega, N.; Millam, J. M.; Klene, M.; Knox, J. E.; Cross, J. B.; Bakken, V.; Adamo, C.; Jaramillo, J.; Gomperts, R.; Stratmann, R. E.; Yazyev, O.; Austin, A. J.; Cammi, R.; Pomelli, C.; Ochterski, J. W.; Martin, R. L.; Morokuma, K.; Zakrzewski, V. G.; Voth, G. A.; P. Salvador, J. J. D.; Dapprich, S.; Daniels, A. D.; Farkas, O.; Foresman, J. B.; Ortiz, J. V.; Cioslowski, J.; Fox, D. J.; Gaussian 09, Revision A.02; Gaussian, Inc, Wallingford, 2009.

53. Hohenberg, P.; Kohn, W.; Phys. Rev. 1964, 136, B864.

54. Becke, A. D.; J. Chem. Phys. 1993, 98, 5648.

55. Ditchfield, R.; Hehre, W. J.; Pople, J. A.; J. Chem. Phys. 1971, 54, 724.

56. Mardirossian, N.; Head-Gordon, M.; Mol. Phys. 2017, 115, 2315.

57. Dean, J. A.; Lange's Handbook of Chemistry, $15^{\text {th }}$ ed., McGraw-Hill, 1999.

58. Hehre, W. J.; Deppmeier, B. J.; Klunzinger, P. E.; Chem. Eng. News 1999, 77, 2 .

59. Lu, T.; Chen, F.; J. Comput. Chem. 2012, 33, 580.

60. Solomons, T. W. G.; Fryhle, C. B.; Química Orgânica Vol 2, 10ª ed., LTC: Rio de Janeiro, 2015.

61. Møller, C.; Plesset, M. S.; Phys. Rev. 1934, 46, 618. 